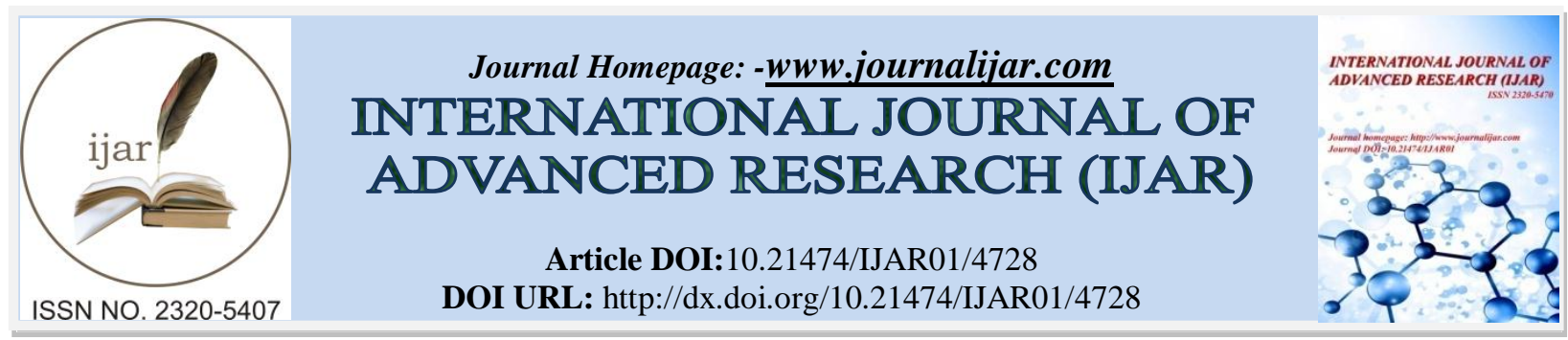

RESEARCH ARTICLE

\title{
AVIFAUNAL DIVERSITY IN AND AROUND BHASKEL DAM RESERVOIR OF NABARANGPUR, ODISHA.
}

\begin{abstract}
"Pramod Pal ${ }^{1}$, Abhilash Acharya ${ }^{1}$ and Hemanta Kumar Sahu ${ }^{2}$.
1. M.Phil student, Department of Zoology, North Orissa University, Takatpur, Baripada, Odisha, India.

2. Associate Professor, Department of Zoology, North Orissa University, Takatpur, Baripada, Odisha, India.
\end{abstract}

\section{Manuscript Info}

Manuscript History

Received: 4 May 2017

Final Accepted: 6 June 2017

Published: July 2017

Key words:-

Avifauna, species, genera, Shanon-

Weiner diversity index, Simpson

diversity index, Bhaskel Dam

\section{Abstract}

Birds are distributed all over the world occupying various habitats. Birds are important bio-indicators of nature so monitoring bird population is important. India's biodiversity is very rich with many types of rare flora and fauna. The present study has been conducted to record the avifaunal diversity in and around the Bhaskel Dam reservoir in the Nabarangpur district of Odisha. Though many varieties of birds are seen in and around the dam no study has been conducted so far on the bird diversity of the area. After a study of about one year a total of $\mathbf{3 , 6 3 3}$ birds belonging to 150 species, 115 genera, 56 families and 19 orders were recorded. The Shanon-Weiner's diversity index was found to be $\mathbf{4 . 7 2 4}$ and the Simpson's diversity index was found to be 0.99 which signifies a good avifaunal diversity in the study area.

Copy Right, IJAR, 2017,. All rights reserved.

\section{Introduction:-}

Birds are one of the most successful group of organisms found on earth. These warm blooded vertebrates have adapted to a wide range of environmental conditions and they occupy diverse habitats. They are found on all the seven continents, including Antarctica. Birds play very important roles in various types of ecosystem as predators, scavengers, pollinators, seed dispersers and they are an important part of various food webs found in nature. Birds are ideal bio-indicators and useful models for studying a variety of environmental problems (Newton, 1995). Out of the 9,000 species of birds under 75 families found globally, India accounts for more than 1300 species under 48 families in 10 bio-geographic zones (Ali and Ripley, 1987). There are about 479 species of birds which are found in Odisha (Dev, 1997). The avifauna of Odisha has been mainly studied by Mukherjee (1952), Ripley (1979), Abdulali (1984), Sahu \& Kar (1999), Sahu \& Rout (2005), Gopi \& Pandav (2007), and Das et al. (2010). Inspite of various studies conducted on avifaunal diversity some remote places of the state like the present study area does not have any specific records of bird species which are found here and therefore no conservation or public awareness initiatives are being taken.

The area where the present study has been conducted i.e., the Bhaskel Dam reservoir of Nabarangpur district of Odisha is a picnic spot and has forested areas as well as woodland regions around them throughout, a variety of small mammals, reptiles and birds can be seen here. Though many varieties of birds are seen in and around the dam no study has been conducted so far on the avifaunal diversity of 
the area and no steps have been taken in the conservation of the natural habitat around this dam. The present study has therefore been conducted to know the bird diversity and to create awareness about the importance of the study area.

\section{Materials and Methods:-}

\section{Study area:-}

The area where the present study has been conducted is the Bhaskel Dam reservoir (Latitude-19 $-42^{\prime}-30^{\prime \prime} \mathrm{N}$ and Longitude- $82^{\circ}-08^{\prime}-00^{\prime \prime} \mathrm{E}$ ) which is located in the Umerkote town of Nabarangpur district of the state of Odisha. The dam is about $1535 \mathrm{M}$ long and about $22.86 \mathrm{M}$ high. It has been build on the river Bhaskel which flows through Nabarangpur district. The dam is mainly used for irrigation and pisciculture. Bhaskel Dam reservoir is a place of tourist attraction and draws many people every year due to its beautiful surrounding which is covered with lush green vegetation. The climate is sub tropical to temperate. It is charcterised by hot and dry summer, cool and humid monsoon and cold and dry winter. December is the coldest month with mean daily average temperature of $25^{\circ} \mathrm{C}$ which reaches to a maximum of up to $40^{\circ} \mathrm{C}$ in May. The rainfall this area receives is mainly from the Southwest monsoons which lasts from June to October. The average annual rainfall varies from $1030.21 \mathrm{~mm}$ to $1569.50 \mathrm{~mm}$. Two types of soils are mainly found in the area i.e., Red and Laterite soil. The soil $\mathrm{pH}$ is neutral to alkaline and its salinity is mainly normal. The vegetation around the study area is mainly of three types; dry mixed deciduous forest, dry peninsular dry forest and dry teak forest. The flora of the study area is predominated by plants like Tamarind (Tamarindus indica), Kusum (Schleichera oleosa), Sal (Shorea robusta), Teak (Tectona grandis), Kendu (Diospyros melanoxylon), Kadamba (Neolamarckia cadamba), Amla (Phyllanthus emblica), Jamun (Syzygium cumini) and Bamboo (Bambusa vulgaris) etc. The present study is an attempt to record the various species of birds found in this area and to understand the ecological significance of this place.

\section{Methodology:-}

Avifaunal diversity in and around the Bhaskel Dam reservoir was recorded from March 2016 to April 2017. Sampling was carried out for thirteen months to record seasonal variation in avifaunal diversity and vegetation. Regular field trips were made throughout this period to the study area. Visits were carried everyday during all the months of the study period to record the bird diversity. The birds were observed at most active period of the day, i.e., early morning from 06:00 to 09:00 hours and in the evening from 15:00 to 18:00 hours. However the observation was made throughout the day also. Nocturnal species were also recorded during the night time. Binocular Olympus $10 * 50 \mathrm{X}$, was used for close observation of birds. Apart from direct sightings the presence of birds was also confirmed by interviews with local forest staffs, villagers and hunters. The birds were identified and classified on the basis of standard field guides by Ali and Ripley (1987), Ali (2002). The birds checklist was prepared using standardized common and scientific names by Manakadan and Pittie (2001).

\section{Data analysis:-}

Bird diversity was calculated using both Shannon-Weiner and Simpson's diversity indices. Shannon-Weiner diversity Index ' $\mathrm{H}$ ' was calculated using the formula:

$$
H^{\prime}=-\sum_{i=1}^{R} p_{i} \ln p_{i}
$$

Where, $P i=$ Proportion of individual species and $R=$ total number of species of the community (number seen and heard).

Simpson's diversity Index 'D' was calculated using the formula: 


\section{$\mathrm{D}=\frac{\sum \mathrm{n}_{i}\left(\mathrm{n}_{i}-1\right)}{\mathrm{N}(\mathrm{N}-1)}$}

Where, $\mathrm{n} i=$ the total number of birds of each individual species and $\mathrm{N}=$ the total number of birds of all species. The value of $\mathrm{D}$ ranges between 0 and 1 . With this index, 1 represents infinite diversity and 0 , no diversity.

The percentage occurrence of birds in each family was calculated using the following formula.

Percentage Occurrence $=($ No. of species of each Family $\div$ Total no. of different species seen $) \times 100$

\section{Results:-}

A total of 3,633 birds belonging to 150 species, 115 genera, 56 families and 19 orders were recorded during the study period. Of the total birds, $134(89.33 \%)$ species were resident (R) and $16(10.66 \%)$ species were migratory (M) (Fig:2). According to the IUCN red list 96\% ( $\mathrm{n}=144)$ species were listed as Least Concern, 2.66\% $(n=4)$ species were Near Threatened, $0.66 \%(n=1)$ was Vulnerable and $0.66 \%(n=1)$ was Endangered (Fig: 3). The birds were also categorised as common (C) $76.66 \%$ ( $\mathrm{n}=115$ ), uncommon (UC) $14 \%$ $(n=21)$ and rare (R) 9.33\% ( $n=14) \quad($ Fig: 4). Dietary pattern of birds showed that insectivores $36.66 \%$ $(\mathrm{n}=55)$ were dominating bird community followed by Piscivores $14 \% \quad(\mathrm{n}=21)$, omnivores $12 \% \quad(\mathrm{n}=18)$, carnivores $12 \%(\mathrm{n}=18)$, frugivores $11.33 \%(\mathrm{n}=17)$, granivores $8.66 \%(\mathrm{n}=13)$ and nectarivores $4.66 \%(\mathrm{n}=7)$ respectively (Fig: 5). Accipitridae and Ardidae were the most dominant families with 6\% (n=9) species followed by Muscicapidae 5.33\% ( $\mathrm{n}=8)$ species, Columbidae 4.66\% ( $\mathrm{n}=7)$ Motacillidae, Strigidae and Sturnidae 4\% ( $\mathrm{n}=6)$ etc (Table 2). To measure the avifaunal diversity both Shanon-Weiner's and Simpson's diversity indices were calculated. The Shanon-Weiner's diversity index was found to be $\mathbf{4 . 7 2 4}$ and the Simpson's diversity index was found to be $\mathbf{0 . 9 9}$ which signifies a good avifaunal diversity in the study area.

\section{Discussion:-}

Most of the bird species recorded were common however some rare species like the Brown fish Owl, Indian eagle Owl, Steppe Eagle, Pallid Harrier, Green Munia, Grey Francolin and Black headed Ibis etc. were recorded around the Bhaskel Dam reservoir. The Steppe Eagle which is an endangered bird was seen only once, the Brown fish Owl was also seen once during the study period so further study should be conducted to know about the status of these birds in the area. Apart from the species recorded sporadic reports of Vultures also occurred though no direct sightings happened during the study period so further investigation is required to know the present status of Vultures and which species if any are present in the area.

Apart from this the breeding and nesting status of birds along with the successful fledging rate is unknown. The attitude of the local human population towards the faunal diversity and their interaction with nature need to be better understood by further investigation. 
Table 1:-Checklist of Birds in and around Bhaskel Dam Reservoir

\begin{tabular}{|c|c|c|c|c|c|c|c|}
\hline $\begin{array}{l}\text { SL } \\
\text { NO }\end{array}$ & FAMILY & SC. NAME & $\begin{array}{l}\text { COMMON } \\
\text { NAME } \\
\end{array}$ & $\begin{array}{l}\text { FEEDING } \\
\text { HABIT }\end{array}$ & ABUNDANCE & STATUS & $\begin{array}{l}\text { IUCN } \\
\text { CATEGORY }\end{array}$ \\
\hline 1 & Podicipedidae & $\begin{array}{l}\text { Tachybaptus } \\
\text { ruficollis }\end{array}$ & Little Grebe & $\mathrm{P}$ & $\mathrm{C}$ & $\mathrm{R}$ & $\begin{array}{l}\text { Least } \\
\text { Concern }\end{array}$ \\
\hline 2 & Anatidae & $\begin{array}{l}\text { Dendrocygna } \\
\text { javanica }\end{array}$ & $\begin{array}{l}\text { Lesser } \\
\text { whistling } \\
\text { Duck }\end{array}$ & $\mathrm{P}$ & $\mathrm{C}$ & $\mathrm{M}$ & $\begin{array}{l}\text { Least } \\
\text { Concern }\end{array}$ \\
\hline 3 & Anatidae & $\begin{array}{l}\text { Nettapus } \\
\text { coromandelinus }\end{array}$ & $\begin{array}{l}\text { Cotton } \\
\text { pygmy } \\
\text { Goose }\end{array}$ & $\mathrm{P}$ & $\mathrm{C}$ & $\mathrm{R}$ & $\begin{array}{l}\text { Least } \\
\text { Concern }\end{array}$ \\
\hline 4 & Rallidae & $\begin{array}{l}\text { Amaurornis } \\
\text { phoenicurus }\end{array}$ & $\begin{array}{l}\text { White } \\
\text { breasted } \\
\text { Water Hen }\end{array}$ & $\mathrm{I}, \mathrm{P}$ & $\mathrm{C}$ & $\mathrm{R}$ & $\begin{array}{l}\text { Least } \\
\text { Concern }\end{array}$ \\
\hline 5 & Rallidae & $\begin{array}{l}\text { Gallinule } \\
\text { chloropus }\end{array}$ & $\begin{array}{l}\text { Common } \\
\text { Moorhen }\end{array}$ & $\mathrm{I}$ & $\mathrm{C}$ & $\mathrm{R}$ & $\begin{array}{l}\text { Least } \\
\text { Concern }\end{array}$ \\
\hline 6 & Rallidae & $\begin{array}{l}\text { Porphyrio } \\
\text { porphyrio }\end{array}$ & $\begin{array}{l}\text { Purple } \\
\text { Moorhen }\end{array}$ & $\mathrm{I}$ & $\mathrm{UC}$ & $\mathrm{R}$ & $\begin{array}{l}\text { Least } \\
\text { Concern }\end{array}$ \\
\hline 7 & Rallidae & Fulica atra & $\begin{array}{l}\text { Common } \\
\text { Coot }\end{array}$ & $\mathrm{O}$ & $\mathrm{C}$ & $\mathrm{M}$ & $\begin{array}{l}\text { Least } \\
\text { Concern }\end{array}$ \\
\hline 8 & Jacanidae & $\begin{array}{l}\text { Hydrophasianus } \\
\text { chirurgus }\end{array}$ & $\begin{array}{l}\text { Pheasant } \\
\text { tailed Jacana }\end{array}$ & $\mathrm{O}$ & $\mathrm{C}$ & $\mathrm{R}$ & $\begin{array}{l}\text { Least } \\
\text { Concern }\end{array}$ \\
\hline 9 & Jacanidae & $\begin{array}{l}\text { Metopidius } \\
\text { indicus }\end{array}$ & $\begin{array}{l}\text { Bronze } \\
\text { winged } \\
\text { Jacana }\end{array}$ & $\mathrm{O}$ & $\mathrm{C}$ & $\mathrm{R}$ & $\begin{array}{l}\text { Least } \\
\text { Concern }\end{array}$ \\
\hline 10 & Charadriidae & $\begin{array}{l}\text { Vanellus } \\
\text { duvauclii }\end{array}$ & $\begin{array}{l}\text { River } \\
\text { Lapwing }\end{array}$ & $\mathrm{I}$ & $\mathrm{UC}$ & $\mathrm{M}$ & $\begin{array}{l}\text { Near } \\
\text { Threatened }\end{array}$ \\
\hline 11 & Charadriidae & Vanellus indicus & $\begin{array}{l}\text { Red wattled } \\
\text { Lapwing }\end{array}$ & $\mathrm{I}$ & $\mathrm{C}$ & $\mathrm{R}$ & $\begin{array}{l}\text { Least } \\
\text { Concern }\end{array}$ \\
\hline 12 & Charadriidae & $\begin{array}{l}\text { Vanellus } \\
\text { malabaricus }\end{array}$ & $\begin{array}{l}\text { Yellow } \\
\text { wattled } \\
\text { Lapwing }\end{array}$ & $\mathrm{I}$ & $\mathrm{UC}$ & $\mathrm{R}$ & $\begin{array}{l}\text { Least } \\
\text { Concern }\end{array}$ \\
\hline 13 & Phalacrocoracidae & Microcarbo niger & $\begin{array}{l}\text { Little } \\
\text { Cormorant }\end{array}$ & $\mathrm{P}$ & $\mathrm{C}$ & $\mathrm{R}$ & $\begin{array}{l}\text { Least } \\
\text { Concern }\end{array}$ \\
\hline 14 & Phalacrocoracidae & $\begin{array}{l}\text { Phalacrocorx } \\
\text { fuscicollis }\end{array}$ & $\begin{array}{l}\text { Indian } \\
\text { Cormorant }\end{array}$ & $\mathrm{P}$ & $\mathrm{C}$ & $\mathrm{R}$ & $\begin{array}{l}\text { Least } \\
\text { Concern }\end{array}$ \\
\hline 15 & Ardeidae & $\begin{array}{l}\text { Ixobrychus } \\
\text { sinensis }\end{array}$ & $\begin{array}{l}\text { Yellow } \\
\text { Bittern }\end{array}$ & $\mathrm{I}, \mathrm{P}$ & $\mathrm{C}$ & $\mathrm{R}$ & $\begin{array}{l}\text { Least } \\
\text { Concern }\end{array}$ \\
\hline 16 & Ardeidae & $\begin{array}{l}\text { Ixobrychus } \\
\text { cinnamomeus }\end{array}$ & $\begin{array}{l}\text { Cinnamon } \\
\text { Bittern } \\
\end{array}$ & $\mathrm{P}$ & $\mathrm{C}$ & $\mathrm{R}$ & $\begin{array}{l}\text { Least } \\
\text { Concern }\end{array}$ \\
\hline 17 & Ardeidae & $\begin{array}{l}\text { Ixobrychus } \\
\text { flavicollis }\end{array}$ & $\begin{array}{l}\text { Black } \\
\text { Bittern }\end{array}$ & $\mathrm{I}, \mathrm{P}$ & $\mathrm{C}$ & $\mathrm{R}$ & $\begin{array}{l}\text { Least } \\
\text { Concern }\end{array}$ \\
\hline 18 & Ardeidae & $\begin{array}{l}\text { Nycticorax } \\
\text { nycticorax }\end{array}$ & $\begin{array}{l}\text { Black } \\
\text { crowned } \\
\text { night Heron }\end{array}$ & $\mathrm{P}$ & $\mathrm{C}$ & $\mathrm{R}$ & $\begin{array}{l}\text { Least } \\
\text { Concern }\end{array}$ \\
\hline 19 & Ardeidae & Ardeola grayii & $\begin{array}{l}\text { Indian pond } \\
\text { Heron }\end{array}$ & $\mathrm{I}, \mathrm{P}$ & $\mathrm{C}$ & $\mathrm{R}$ & $\begin{array}{l}\text { Least } \\
\text { Concern }\end{array}$ \\
\hline 20 & Ardeidae & Bubulcus ibis & Cattle Egret & $\mathrm{I}, \mathrm{P}$ & $\mathrm{C}$ & $\mathrm{R}$ & $\begin{array}{l}\text { Least } \\
\text { Concern }\end{array}$ \\
\hline 21 & Ardeidae & Egretta garzetta & Little Egret & $\mathrm{I}, \mathrm{P}$ & $\mathrm{C}$ & $\mathrm{R}$ & $\begin{array}{l}\text { Least } \\
\text { Concern }\end{array}$ \\
\hline 22 & Ardeidae & $\begin{array}{l}\text { Mesophoyx } \\
\text { intermedia }\end{array}$ & $\begin{array}{l}\text { Intermediate } \\
\text { Egret }\end{array}$ & $\mathrm{I}, \mathrm{P}$ & $\mathrm{C}$ & $\mathrm{R}$ & $\begin{array}{l}\text { Least } \\
\text { Concern }\end{array}$ \\
\hline 23 & Ardeidae & Ardea alba & Great Egret & $\mathrm{I}, \mathrm{P}$ & $\mathrm{UC}$ & $\mathrm{R}$ & $\begin{array}{l}\text { Least } \\
\text { Concern }\end{array}$ \\
\hline
\end{tabular}




\begin{tabular}{|c|c|c|c|c|c|c|c|}
\hline 24 & Ciconiidae & $\begin{array}{l}\text { Anastomus } \\
\text { oscitans }\end{array}$ & $\begin{array}{l}\text { Open billed } \\
\text { Stork }\end{array}$ & $\mathrm{P}$ & $\mathrm{C}$ & $\mathrm{R}$ & $\begin{array}{l}\text { Least } \\
\text { Concern }\end{array}$ \\
\hline 25 & Threskiornithidae & $\begin{array}{l}\text { Threskiornis } \\
\text { melanocephalus }\end{array}$ & $\begin{array}{l}\text { Black } \\
\text { headed Ibis }\end{array}$ & $\mathrm{I}, \mathrm{P}$ & UC & M & $\begin{array}{l}\text { Near } \\
\text { Threatened }\end{array}$ \\
\hline 26 & Columbidae & Treron bicinceus & $\begin{array}{l}\text { Orange } \\
\text { breasted } \\
\text { Green } \\
\text { pigeon }\end{array}$ & $\mathrm{F}$ & $\mathrm{UC}$ & $\mathrm{R}$ & $\begin{array}{l}\text { Least } \\
\text { Concern }\end{array}$ \\
\hline 27 & Columbidae & $\begin{array}{l}\text { Treron } \\
\text { phoenicoptera }\end{array}$ & $\begin{array}{l}\text { Yellow } \\
\text { legged } \\
\text { Green } \\
\text { Pigeon }\end{array}$ & $\mathrm{F}$ & $\mathrm{C}$ & $\mathrm{R}$ & $\begin{array}{l}\text { Least } \\
\text { Concern }\end{array}$ \\
\hline 28 & Columbidae & $\begin{array}{l}\text { Streptopilia } \\
\text { orientalis }\end{array}$ & $\begin{array}{l}\text { Oriental } \\
\text { Turtle Dove }\end{array}$ & $\mathrm{G}$ & $\mathrm{C}$ & $\mathrm{R}$ & $\begin{array}{l}\text { Least } \\
\text { Concern }\end{array}$ \\
\hline 29 & Columbidae & $\begin{array}{l}\text { Spilopilia } \\
\text { chinensis }\end{array}$ & $\begin{array}{l}\text { Spotted } \\
\text { Dove }\end{array}$ & G & $\mathrm{C}$ & $\mathrm{R}$ & $\begin{array}{l}\text { Least } \\
\text { Concern }\end{array}$ \\
\hline 30 & Columbidae & $\begin{array}{l}\text { Streptopilia } \\
\text { decaocto }\end{array}$ & $\begin{array}{l}\text { Eurasian } \\
\text { Collared } \\
\text { Dove }\end{array}$ & G & $\mathrm{C}$ & $\mathrm{R}$ & $\begin{array}{l}\text { Least } \\
\text { Concern }\end{array}$ \\
\hline 31 & Columbidae & $\begin{array}{l}\text { Chalcophaps } \\
\text { indica }\end{array}$ & $\begin{array}{l}\text { Emerald } \\
\text { Dove }\end{array}$ & $\mathrm{G}$ & $\mathrm{C}$ & $\mathrm{R}$ & $\begin{array}{l}\text { Least } \\
\text { Concern }\end{array}$ \\
\hline 32 & Columbidae & Columba livia & $\begin{array}{l}\text { Common } \\
\text { Rock Pigeon }\end{array}$ & G & $\mathrm{C}$ & $\mathrm{R}$ & $\begin{array}{l}\text { Least } \\
\text { Concern }\end{array}$ \\
\hline 33 & Psittaculidae & $\begin{array}{l}\text { Psittacula } \\
\text { eupatria }\end{array}$ & $\begin{array}{l}\text { Alexandrine } \\
\text { Parakeet }\end{array}$ & $\mathrm{F}$ & $\mathrm{C}$ & $\mathrm{R}$ & $\begin{array}{l}\text { Near } \\
\text { Threatened }\end{array}$ \\
\hline 34 & Psittaculidae & $\begin{array}{l}\text { Psittacula } \\
\text { krameri }\end{array}$ & $\begin{array}{l}\text { Rose ringed } \\
\text { Parakeet }\end{array}$ & $\mathrm{F}$ & $\mathrm{C}$ & $\mathrm{R}$ & $\begin{array}{l}\text { Least } \\
\text { Concern }\end{array}$ \\
\hline 35 & Psittaculidae & $\begin{array}{l}\text { Psittacula } \\
\text { cyanocephala }\end{array}$ & $\begin{array}{l}\text { Plum headed } \\
\text { Parakeet }\end{array}$ & $\mathrm{F}$ & $\mathrm{C}$ & $\mathrm{R}$ & $\begin{array}{l}\text { Least } \\
\text { Concern }\end{array}$ \\
\hline 36 & Cuculidae & $\begin{array}{l}\text { Clamator } \\
\text { jacobinus }\end{array}$ & Pied Cuckoo & I & $\mathrm{C}$ & $\mathrm{M}$ & $\begin{array}{l}\text { Least } \\
\text { Concern }\end{array}$ \\
\hline 37 & Cuculidae & $\begin{array}{l}\text { Hierococcyx } \\
\text { varius }\end{array}$ & $\begin{array}{l}\text { Common } \\
\text { Hawk } \\
\text { Cuckoo }\end{array}$ & I & $\mathrm{C}$ & $\mathrm{R}$ & $\begin{array}{l}\text { Least } \\
\text { Concern }\end{array}$ \\
\hline 38 & Cuculidae & $\begin{array}{l}\text { Eudynamys } \\
\text { scolopaceus }\end{array}$ & Indian Koel & $\mathrm{F}$ & $\mathrm{C}$ & $\mathrm{R}$ & $\begin{array}{l}\text { Least } \\
\text { Concern }\end{array}$ \\
\hline 39 & Cuculidae & $\begin{array}{l}\text { Phaenicophas } \\
\text { leschenaultii }\end{array}$ & $\begin{array}{l}\text { Sirkeer } \\
\text { Malhoka }\end{array}$ & I & UC & $\mathrm{R}$ & $\begin{array}{l}\text { Least } \\
\text { Concern }\end{array}$ \\
\hline 40 & Cuculidae & $\begin{array}{l}\text { Centropus } \\
\text { sinensis }\end{array}$ & $\begin{array}{l}\text { Greater } \\
\text { Coucal }\end{array}$ & $\mathrm{O}$ & $\mathrm{C}$ & $\mathrm{R}$ & $\begin{array}{l}\text { Least } \\
\text { Concern }\end{array}$ \\
\hline 41 & Accipitridae & Milvus migrans & Pariah Kite & $\mathrm{Ca}$ & $\mathrm{C}$ & $\mathrm{R}$ & $\begin{array}{l}\text { Least } \\
\text { Concern }\end{array}$ \\
\hline 42 & Accipitridae & Elanus caeruleus & $\begin{array}{l}\text { Black } \\
\text { Winged Kite }\end{array}$ & $\mathrm{Ca}$ & $\mathrm{C}$ & $\mathrm{R}$ & $\begin{array}{l}\text { Least } \\
\text { Concern }\end{array}$ \\
\hline 43 & Accipitridae & Accipiter badius & Shikra & $\mathrm{Ca}$ & $\mathrm{C}$ & $\mathrm{R}$ & $\begin{array}{l}\text { Least } \\
\text { Concern }\end{array}$ \\
\hline 44 & Accipitridae & $\begin{array}{l}\text { Pernis } \\
\text { ptilorhynchus }\end{array}$ & $\begin{array}{l}\text { Oriental } \\
\text { Honey } \\
\text { Buzard }\end{array}$ & $\mathrm{Ca}$ & $\mathrm{Ra}$ & $\mathrm{R}$ & $\begin{array}{l}\text { Least } \\
\text { Concern }\end{array}$ \\
\hline 45 & Accipitridae & $\begin{array}{l}\text { Circus } \\
\text { melanoleucos }\end{array}$ & Pied Harrier & $\mathrm{Ca}$ & $\mathrm{Ra}$ & $\mathrm{M}$ & $\begin{array}{l}\text { Least } \\
\text { Concern }\end{array}$ \\
\hline 46 & Accipitridae & $\begin{array}{l}\text { Circus } \\
\text { macrourus }\end{array}$ & $\begin{array}{l}\text { Pallid } \\
\text { Harrier }\end{array}$ & $\mathrm{Ca}$ & $\mathrm{Ra}$ & M & $\begin{array}{l}\text { Near } \\
\text { Threatened }\end{array}$ \\
\hline 47 & Accipitridae & Spilornis cheela & $\begin{array}{l}\text { Crested } \\
\text { serpent }\end{array}$ & $\mathrm{Ca}$ & $\mathrm{C}$ & $\mathrm{R}$ & $\begin{array}{l}\text { Least } \\
\text { Concern }\end{array}$ \\
\hline
\end{tabular}




\begin{tabular}{|c|c|c|c|c|c|c|c|}
\hline & & & Eagle & & & & \\
\hline 48 & Accipitridae & $\begin{array}{l}\text { Circaetus } \\
\text { gallicus }\end{array}$ & $\begin{array}{l}\text { Short toed } \\
\text { Eagle }\end{array}$ & $\mathrm{Ca}$ & $\mathrm{C}$ & $\mathrm{R}$ & $\begin{array}{l}\text { Least } \\
\text { Concern }\end{array}$ \\
\hline 49 & Accipitridae & Aquila nipalensis & $\begin{array}{l}\text { Steppe } \\
\text { Eagle }\end{array}$ & $\mathrm{Ca}$ & $\mathrm{Ra}$ & M & Endangered \\
\hline 50 & Falconidae & $\begin{array}{l}\text { Falco } \\
\text { tinnunculus }\end{array}$ & $\begin{array}{l}\text { Common } \\
\text { Kestrel }\end{array}$ & $\mathrm{Ca}$ & $\mathrm{C}$ & M & $\begin{array}{l}\text { Least } \\
\text { Concern }\end{array}$ \\
\hline 51 & Falconidae & Falco peregrinus & $\begin{array}{l}\text { Peregrine } \\
\text { Falcon }\end{array}$ & $\mathrm{Ca}$ & $\mathrm{UC}$ & $\mathrm{M}$ & $\begin{array}{l}\text { Least } \\
\text { Concern }\end{array}$ \\
\hline 52 & Strigidae & Otus lettia & $\begin{array}{l}\text { Collared } \\
\text { Scops Owl }\end{array}$ & $\mathrm{Ca}$ & $\mathrm{C}$ & $\mathrm{R}$ & $\begin{array}{l}\text { Least } \\
\text { Concern }\end{array}$ \\
\hline 53 & Strigidae & $\begin{array}{l}\text { Glaucidium } \\
\text { radiatum }\end{array}$ & $\begin{array}{l}\text { Barred } \\
\text { Jungle } \\
\text { Owlet }\end{array}$ & $\mathrm{Ca}$ & $\mathrm{Ra}$ & $\mathrm{R}$ & $\begin{array}{l}\text { Least } \\
\text { Concern }\end{array}$ \\
\hline 54 & Strigidae & Ninox scutulata & $\begin{array}{l}\text { Brown } \\
\text { Hawk Owl }\end{array}$ & $\mathrm{Ca}$ & $\mathrm{Ra}$ & $\mathrm{R}$ & $\begin{array}{l}\text { Least } \\
\text { Concern }\end{array}$ \\
\hline 55 & Strigidae & Athene brama & $\begin{array}{l}\text { Spotted } \\
\text { Owlet }\end{array}$ & $\mathrm{Ca}, \mathrm{I}$ & $\mathrm{C}$ & $\mathrm{R}$ & $\begin{array}{l}\text { Least } \\
\text { Concern }\end{array}$ \\
\hline 56 & Strigidae & Bubo zeylonensis & $\begin{array}{l}\text { Brown fish } \\
\text { Owl }\end{array}$ & $\mathrm{Ca}, \mathrm{P}$ & $\mathrm{Ra}$ & $\mathrm{R}$ & $\begin{array}{l}\text { Least } \\
\text { Concern }\end{array}$ \\
\hline 57 & Strigidae & Bubo bengalensis & $\begin{array}{l}\text { Indian Eagle } \\
\text { Owl }\end{array}$ & $\mathrm{Ca}$ & $\mathrm{Ra}$ & $\mathrm{R}$ & $\begin{array}{l}\text { Least } \\
\text { Concern }\end{array}$ \\
\hline 58 & Tytonidae & Tyto alba & Barn Owl & $\mathrm{Ca}$ & $\mathrm{C}$ & $\mathrm{R}$ & $\begin{array}{l}\text { Least } \\
\text { Concern }\end{array}$ \\
\hline 59 & Caprimuligidae & $\begin{array}{l}\text { Caprimulgus } \\
\text { asiaticus }\end{array}$ & $\begin{array}{l}\text { Indian } \\
\text { Nightjar }\end{array}$ & I & $\mathrm{C}$ & $\mathrm{R}$ & $\begin{array}{l}\text { Least } \\
\text { Concern }\end{array}$ \\
\hline 60 & Caprimuligidae & $\begin{array}{l}\text { Caprimulgus } \\
\text { indicus }\end{array}$ & $\begin{array}{l}\text { Jungle } \\
\text { Nightjar }\end{array}$ & I & $\mathrm{C}$ & $\mathrm{R}$ & $\begin{array}{l}\text { Least } \\
\text { Concern }\end{array}$ \\
\hline 61 & Alcedinidae & Alcedo atthis & $\begin{array}{l}\text { Small Blue } \\
\text { kingfisher }\end{array}$ & $\mathrm{P}$ & $\mathrm{C}$ & $\mathrm{R}$ & $\begin{array}{l}\text { Least } \\
\text { Concern }\end{array}$ \\
\hline 62 & Alcedinidae & $\begin{array}{l}\text { Halcyon } \\
\text { smyrnensis }\end{array}$ & $\begin{array}{l}\text { White } \\
\text { breasted } \\
\text { kingfisher }\end{array}$ & $\mathrm{P}$ & $\mathrm{C}$ & $\mathrm{R}$ & $\begin{array}{l}\text { Least } \\
\text { Concern }\end{array}$ \\
\hline 63 & Alcedinidae & Ceryle rudis & $\begin{array}{l}\text { Pied } \\
\text { kingfisher }\end{array}$ & $\mathrm{P}$ & UC & $\mathrm{R}$ & $\begin{array}{l}\text { Least } \\
\text { Concern }\end{array}$ \\
\hline 64 & Alcedinidae & $\begin{array}{l}\text { Pelargopsis } \\
\text { capensis }\end{array}$ & $\begin{array}{l}\text { Stork billed } \\
\text { kingfisher }\end{array}$ & $\mathrm{P}$ & $\mathrm{UC}$ & $\mathrm{R}$ & $\begin{array}{l}\text { Least } \\
\text { Concern }\end{array}$ \\
\hline 65 & Meropidae & Merops orientalis & $\begin{array}{l}\text { Common } \\
\text { Bee eater }\end{array}$ & I & $\mathrm{C}$ & $\mathrm{R}$ & $\begin{array}{l}\text { Least } \\
\text { Concern }\end{array}$ \\
\hline 66 & Meropidae & $\begin{array}{l}\text { Merops } \\
\text { leschenaultia }\end{array}$ & $\begin{array}{l}\text { Chestnut } \\
\text { headed Bee } \\
\text { eater }\end{array}$ & I & $\mathrm{C}$ & $\mathrm{R}$ & $\begin{array}{l}\text { Least } \\
\text { Concern }\end{array}$ \\
\hline 67 & Meropidae & $\begin{array}{l}\text { Merops } \\
\text { philippinus }\end{array}$ & $\begin{array}{l}\text { Blue tailed } \\
\text { Bee eater }\end{array}$ & $\mathrm{I}$ & $\mathrm{C}$ & $\mathrm{M}$ & $\begin{array}{l}\text { Least } \\
\text { Concern } \\
\end{array}$ \\
\hline 68 & Megalaimidae & $\begin{array}{l}\text { Psilopogon } \\
\text { zeylanicus }\end{array}$ & $\begin{array}{l}\text { Brown } \\
\text { headed } \\
\text { Barbet }\end{array}$ & $\mathrm{F}$ & $\mathrm{C}$ & $\mathrm{R}$ & $\begin{array}{l}\text { Least } \\
\text { Concern }\end{array}$ \\
\hline 69 & Megalaimidae & $\begin{array}{l}\text { Psilopogon } \\
\text { asiaticus }\end{array}$ & $\begin{array}{l}\text { Blue } \\
\text { throated } \\
\text { Barbet }\end{array}$ & $\mathrm{F}$ & $\mathrm{C}$ & $\mathrm{R}$ & $\begin{array}{l}\text { Least } \\
\text { Concern }\end{array}$ \\
\hline 70 & Megalaimidae & $\begin{array}{l}\text { Psilopogon } \\
\text { haemacephalus }\end{array}$ & $\begin{array}{l}\text { Coppersmith } \\
\text { Barbet }\end{array}$ & $\mathrm{F}$ & $\mathrm{C}$ & $\mathrm{R}$ & $\begin{array}{l}\text { Least } \\
\text { Concern }\end{array}$ \\
\hline 71 & Coraciidae & $\begin{array}{l}\text { Coracias } \\
\text { benghalensis }\end{array}$ & $\begin{array}{l}\text { Indian } \\
\text { Roller }\end{array}$ & I & $\mathrm{C}$ & $\mathrm{R}$ & $\begin{array}{l}\text { Least } \\
\text { Concern } \\
\end{array}$ \\
\hline 72 & Upupidae & Upupa epops & Common & I & $\mathrm{C}$ & $\mathrm{R}$ & Least \\
\hline
\end{tabular}




\begin{tabular}{|c|c|c|c|c|c|c|c|}
\hline & & & Hoopoe & & & & Concern \\
\hline 73 & Picidae & Jynx torquilla & $\begin{array}{l}\text { European } \\
\text { Wryneck }\end{array}$ & I & UC & $\mathrm{R}$ & $\begin{array}{l}\text { Least } \\
\text { Concern }\end{array}$ \\
\hline 74 & Picidae & $\begin{array}{l}\text { Dinopium } \\
\text { benghalense }\end{array}$ & $\begin{array}{l}\text { Black } \\
\text { rumped } \\
\text { Flameback }\end{array}$ & F,I & $\mathrm{C}$ & $\mathrm{R}$ & $\begin{array}{l}\text { Least } \\
\text { Concern }\end{array}$ \\
\hline 75 & Picidae & $\begin{array}{l}\text { Dendrocopos } \\
\text { macei }\end{array}$ & $\begin{array}{l}\text { Fulvous } \\
\text { breasted } \\
\text { Woodpecker }\end{array}$ & F,I & $\mathrm{C}$ & $\mathrm{R}$ & $\begin{array}{l}\text { Least } \\
\text { Concern }\end{array}$ \\
\hline 76 & Picidae & $\begin{array}{l}\text { Leiopicus } \\
\text { marattensis }\end{array}$ & $\begin{array}{l}\text { Yellow } \\
\text { fronted Pied } \\
\text { Woodpecker }\end{array}$ & F,I & $\mathrm{UC}$ & $\mathrm{R}$ & $\begin{array}{l}\text { Least } \\
\text { Concern }\end{array}$ \\
\hline 77 & Picidae & $\begin{array}{l}\text { Chrysocolaptes } \\
\text { guttacristatus }\end{array}$ & $\begin{array}{l}\text { Large } \\
\text { Flameback } \\
\text { Woodpecker }\end{array}$ & F,I & $\mathrm{C}$ & $\mathrm{R}$ & $\begin{array}{l}\text { Least } \\
\text { Concern }\end{array}$ \\
\hline 78 & Bucerotidae & $\begin{array}{l}\text { Anthracoceros } \\
\text { albirostris }\end{array}$ & $\begin{array}{l}\text { Pied } \\
\text { Hornbill }\end{array}$ & F,I & $\mathrm{C}$ & $\mathrm{R}$ & $\begin{array}{l}\text { Least } \\
\text { Concern }\end{array}$ \\
\hline 79 & Bucerotidae & $\begin{array}{l}\text { Ocyceros } \\
\text { birostris } \\
\end{array}$ & $\begin{array}{l}\text { Indian Grey } \\
\text { Hornbill }\end{array}$ & F,I & $\mathrm{C}$ & $\mathrm{R}$ & $\begin{array}{l}\text { Least } \\
\text { Concern }\end{array}$ \\
\hline 80 & Hirundinidae & Hirundo rustica & $\begin{array}{l}\text { Barn } \\
\text { Swallow } \\
\end{array}$ & I & $\mathrm{C}$ & $\mathrm{R}$ & $\begin{array}{l}\text { Least } \\
\text { Concern }\end{array}$ \\
\hline 81 & Hirundinidae & Hirundo smithii & $\begin{array}{l}\text { Wire tailed } \\
\text { Swallow }\end{array}$ & I & UC & $\mathrm{R}$ & $\begin{array}{l}\text { Least } \\
\text { Concern }\end{array}$ \\
\hline 82 & Hirundinidae & Cecropis daurica & $\begin{array}{l}\text { Red rumped } \\
\text { Swallow }\end{array}$ & I & $\mathrm{UC}$ & $\mathrm{R}$ & $\begin{array}{l}\text { Least } \\
\text { Concern }\end{array}$ \\
\hline 83 & Hirundinidae & $\begin{array}{l}\text { Cecropis } \\
\text { striolata }\end{array}$ & $\begin{array}{l}\text { Striated } \\
\text { Swallow }\end{array}$ & I & $\mathrm{C}$ & $\mathrm{R}$ & $\begin{array}{l}\text { Least } \\
\text { Concern }\end{array}$ \\
\hline 84 & Alaudidae & $\begin{array}{l}\text { Mirafra } \\
\text { erythroptera }\end{array}$ & $\begin{array}{l}\text { Indian Bush } \\
\text { Lark }\end{array}$ & I & $\mathrm{C}$ & $\mathrm{R}$ & $\begin{array}{l}\text { Least } \\
\text { Concern }\end{array}$ \\
\hline 85 & Timaliidae & $\begin{array}{l}\text { Pteruthius } \\
\text { rufiventer }\end{array}$ & $\begin{array}{l}\text { Black } \\
\text { headed } \\
\text { Shrike }\end{array}$ & I & $\mathrm{C}$ & $\mathrm{R}$ & $\begin{array}{l}\text { Least } \\
\text { Concern }\end{array}$ \\
\hline 86 & Timaliidae & $\begin{array}{l}\text { Dumetia } \\
\text { hyperythra }\end{array}$ & $\begin{array}{l}\text { Rufous } \\
\text { bellied } \\
\text { Babbler }\end{array}$ & I & $\mathrm{C}$ & $\mathrm{R}$ & $\begin{array}{l}\text { Least } \\
\text { Concern }\end{array}$ \\
\hline 87 & Dicruridae & $\begin{array}{l}\text { Dicrusus } \\
\text { macrocercus }\end{array}$ & $\begin{array}{l}\text { Black } \\
\text { Drongo }\end{array}$ & I & $\mathrm{C}$ & $\mathrm{R}$ & $\begin{array}{l}\text { Least } \\
\text { Concern }\end{array}$ \\
\hline 88 & Dicruridae & $\begin{array}{l}\text { Dicrusus } \\
\text { caerulescens }\end{array}$ & $\begin{array}{l}\text { White } \\
\text { bellied } \\
\text { Drongo }\end{array}$ & I & $\mathrm{C}$ & $\mathrm{R}$ & $\begin{array}{l}\text { Least } \\
\text { Concern }\end{array}$ \\
\hline 89 & Dicruridae & $\begin{array}{l}\text { Dicrurus } \\
\text { hottentottus }\end{array}$ & $\begin{array}{l}\text { Hair crested } \\
\text { Drongo }\end{array}$ & I & $\mathrm{C}$ & $\mathrm{R}$ & $\begin{array}{l}\text { Least } \\
\text { Concern }\end{array}$ \\
\hline 90 & Dicruridae & $\begin{array}{l}\text { Dicrurus } \\
\text { paradiseus }\end{array}$ & $\begin{array}{l}\text { Racket } \\
\text { tailed } \\
\text { Drongo }\end{array}$ & I & $\mathrm{C}$ & $\mathrm{R}$ & $\begin{array}{l}\text { Least } \\
\text { Concern }\end{array}$ \\
\hline 91 & Sturnidae & $\begin{array}{l}\text { Acrido } \\
\text { theresfuscus }\end{array}$ & Jungle Myna & $\mathrm{O}$ & $\mathrm{C}$ & $\mathrm{R}$ & $\begin{array}{l}\text { Least } \\
\text { Concern }\end{array}$ \\
\hline 92 & Sturnidae & $\begin{array}{l}\text { Acrido } \\
\text { theresginginianus }\end{array}$ & Bank Myna & $\mathrm{O}$ & $\mathrm{C}$ & $\mathrm{R}$ & $\begin{array}{l}\text { Least } \\
\text { Concern }\end{array}$ \\
\hline 93 & Sturnidae & Gracula religiosa & Hill Myna & $\mathrm{O}$ & $\mathrm{UC}$ & $\mathrm{R}$ & $\begin{array}{l}\text { Least } \\
\text { Concern }\end{array}$ \\
\hline 94 & Sturnidae & Gracupica contra & $\begin{array}{l}\text { Asian pied } \\
\text { Starling }\end{array}$ & $\mathrm{O}$ & $\mathrm{C}$ & $\mathrm{R}$ & $\begin{array}{l}\text { Least } \\
\text { Concern }\end{array}$ \\
\hline 95 & Sturnidae & $\begin{array}{l}\text { Sturnia } \\
\text { malabarica }\end{array}$ & $\begin{array}{l}\text { Chestnut } \\
\text { tailed }\end{array}$ & $\mathrm{O}$ & $\mathrm{C}$ & $\mathrm{R}$ & $\begin{array}{l}\text { Least } \\
\text { Concern }\end{array}$ \\
\hline
\end{tabular}




\begin{tabular}{|c|c|c|c|c|c|c|c|}
\hline & & & Starling & & & & \\
\hline 96 & Sturnidae & $\begin{array}{l}\text { Sturnia } \\
\text { pagodarum }\end{array}$ & $\begin{array}{l}\text { Brahminy } \\
\text { Starling }\end{array}$ & $\mathrm{O}$ & $\mathrm{C}$ & $\mathrm{R}$ & $\begin{array}{l}\text { Least } \\
\text { Concern }\end{array}$ \\
\hline 97 & Corvidae & $\begin{array}{l}\text { Dendrocitta } \\
\text { formosae }\end{array}$ & Tree pie & $\mathrm{O}$ & $\mathrm{C}$ & $\mathrm{R}$ & $\begin{array}{l}\text { Least } \\
\text { Concern }\end{array}$ \\
\hline 98 & Corvidae & Corvus splendens & $\begin{array}{l}\text { Common } \\
\text { crow }\end{array}$ & $\mathrm{O}$ & $\mathrm{C}$ & $\mathrm{R}$ & $\begin{array}{l}\text { Least } \\
\text { Concern }\end{array}$ \\
\hline 99 & Corvidae & $\begin{array}{l}\text { Corvus } \\
\text { macrorhynchos }\end{array}$ & Jungle crow & $\mathrm{O}$ & $\mathrm{C}$ & $\mathrm{R}$ & $\begin{array}{l}\text { Least } \\
\text { Concern }\end{array}$ \\
\hline 100 & Tephrodornithidae & $\begin{array}{l}\text { Tephrodornis } \\
\text { pondicerianus }\end{array}$ & $\begin{array}{l}\text { Indian } \\
\text { Woodshrike }\end{array}$ & $\mathrm{O}$ & $\mathrm{C}$ & $\mathrm{R}$ & $\begin{array}{l}\text { Least } \\
\text { Concern }\end{array}$ \\
\hline 101 & Campephagidae & Coracina macei & $\begin{array}{l}\text { Indian large } \\
\text { cuckoo- } \\
\text { shrike }\end{array}$ & I & $\mathrm{UC}$ & $\mathrm{R}$ & $\begin{array}{l}\text { Least } \\
\text { Concern }\end{array}$ \\
\hline 102 & Campephagidae & $\begin{array}{l}\text { Pericrocotus } \\
\text { speciosus }\end{array}$ & $\begin{array}{l}\text { Indian } \\
\text { Scarlet } \\
\text { Minivet }\end{array}$ & I & $\mathrm{C}$ & $\mathrm{R}$ & $\begin{array}{l}\text { Least } \\
\text { Concern }\end{array}$ \\
\hline 103 & Aegithinidae & Aegithina tiphia & $\begin{array}{l}\text { Common } \\
\text { Iora }\end{array}$ & I & $\mathrm{C}$ & $\mathrm{R}$ & $\begin{array}{l}\text { Least } \\
\text { Concern }\end{array}$ \\
\hline 104 & Chloropseidae & $\begin{array}{l}\text { Chloropsis } \\
\text { jerdoni }\end{array}$ & $\begin{array}{l}\text { Jerdon's } \\
\text { Chloropsis }\end{array}$ & I & $\mathrm{C}$ & $\mathrm{R}$ & $\begin{array}{l}\text { Least } \\
\text { Concern }\end{array}$ \\
\hline 105 & Chloropseidae & $\begin{array}{l}\text { Chloropsis } \\
\text { aurifrons }\end{array}$ & $\begin{array}{l}\text { Gold fronted } \\
\text { Chloropsis }\end{array}$ & I & $\mathrm{C}$ & $\mathrm{R}$ & $\begin{array}{l}\text { Least } \\
\text { Concern }\end{array}$ \\
\hline 106 & Pycnonotidae & $\begin{array}{l}\text { Pycnononotus } \\
\text { cafer }\end{array}$ & $\begin{array}{l}\text { Red vented } \\
\text { Bulbul }\end{array}$ & F,G,I & $\mathrm{C}$ & $\mathrm{R}$ & $\begin{array}{l}\text { Least } \\
\text { Concern }\end{array}$ \\
\hline 107 & Pycnonotidae & $\begin{array}{l}\text { Pycnonotus } \\
\text { Jocosus }\end{array}$ & $\begin{array}{l}\text { Red } \\
\text { whiskered } \\
\text { Bulbul }\end{array}$ & $\mathrm{F}, \mathrm{G}, \mathrm{I}$ & $\mathrm{C}$ & $\mathrm{R}$ & $\begin{array}{l}\text { Least } \\
\text { Concern }\end{array}$ \\
\hline 108 & Pycnonotidae & $\begin{array}{l}\text { Pycnonotus } \\
\text { atriceps }\end{array}$ & $\begin{array}{l}\text { Black } \\
\text { headed } \\
\text { Bulbul } \\
\end{array}$ & $\mathrm{F}, \mathrm{G}, \mathrm{I}$ & $\mathrm{C}$ & $\mathrm{R}$ & $\begin{array}{l}\text { Least } \\
\text { Concern }\end{array}$ \\
\hline 109 & Pellorneidae & $\begin{array}{l}\text { Pellorneum } \\
\text { ruficeps }\end{array}$ & $\begin{array}{l}\text { Spotted } \\
\text { Babbler }\end{array}$ & I & $\mathrm{C}$ & $\mathrm{R}$ & $\begin{array}{l}\text { Least } \\
\text { Concern }\end{array}$ \\
\hline 110 & Sylviidae & $\begin{array}{l}\text { Chrysomma } \\
\text { sinense }\end{array}$ & $\begin{array}{l}\text { Yellow eyed } \\
\text { Babbler }\end{array}$ & I & $\mathrm{C}$ & $\mathrm{R}$ & $\begin{array}{l}\text { Least } \\
\text { Concern }\end{array}$ \\
\hline 111 & Leiothrichidae & Turdoides striata & $\begin{array}{l}\text { Jungle } \\
\text { Babbler } \\
\end{array}$ & I & $\mathrm{C}$ & $\mathrm{R}$ & $\begin{array}{l}\text { Least } \\
\text { Concern }\end{array}$ \\
\hline 112 & Muscicapidae & Ficedula parva & $\begin{array}{l}\text { Red breasted } \\
\text { Flycatcher }\end{array}$ & I & $\mathrm{UC}$ & $\mathrm{M}$ & $\begin{array}{l}\text { Least } \\
\text { Concern }\end{array}$ \\
\hline 113 & Muscicapidae & $\begin{array}{l}\text { Cyornis } \\
\text { poliogenys }\end{array}$ & $\begin{array}{l}\text { Brook's } \\
\text { Flycatcher }\end{array}$ & I & $\mathrm{Ra}$ & $\mathrm{R}$ & $\begin{array}{l}\text { Least } \\
\text { Concern }\end{array}$ \\
\hline 114 & Muscicapidae & $\begin{array}{l}\text { Cyornis } \\
\text { rubeculoides }\end{array}$ & $\begin{array}{l}\text { Blue } \\
\text { throated } \\
\text { Flycatcher }\end{array}$ & I & $\mathrm{Ra}$ & $\mathrm{R}$ & $\begin{array}{l}\text { Least } \\
\text { Concern }\end{array}$ \\
\hline 115 & Muscicapidae & $\begin{array}{l}\text { Eumyias } \\
\text { thalassinus }\end{array}$ & $\begin{array}{l}\text { Verditer } \\
\text { Flycatcher }\end{array}$ & $\mathrm{I}, \mathrm{F}$ & $\mathrm{Ra}$ & $\mathrm{R}$ & $\begin{array}{l}\text { Least } \\
\text { Concern }\end{array}$ \\
\hline 116 & Muscicapidae & $\begin{array}{l}\text { Copsychus } \\
\text { saularis }\end{array}$ & $\begin{array}{l}\text { Magpie } \\
\text { Robin }\end{array}$ & $\mathrm{O}$ & $\mathrm{C}$ & $\mathrm{R}$ & $\begin{array}{l}\text { Least } \\
\text { Concern }\end{array}$ \\
\hline 117 & Muscicapidae & $\begin{array}{l}\text { Copsychus } \\
\text { fulicatus }\end{array}$ & $\begin{array}{l}\text { Indian } \\
\text { Robin }\end{array}$ & I & $\mathrm{C}$ & $\mathrm{R}$ & $\begin{array}{l}\text { Least } \\
\text { Concern }\end{array}$ \\
\hline 118 & Muscicapidae & Saxicola caprata & $\begin{array}{l}\text { Pied } \\
\text { Bushchat }\end{array}$ & I & $\mathrm{C}$ & $\mathrm{R}$ & $\begin{array}{l}\text { Least } \\
\text { Concern }\end{array}$ \\
\hline 119 & Muscicapidae & $\begin{array}{l}\text { Copsychus } \\
\text { malabaricus }\end{array}$ & $\begin{array}{l}\text { Indian } \\
\text { Shama }\end{array}$ & I & $\mathrm{C}$ & $\mathrm{R}$ & $\begin{array}{l}\text { Least } \\
\text { Concern }\end{array}$ \\
\hline 120 & Cisticolidae & Prinia socialis & Ashy Grey & I & $\mathrm{C}$ & $\mathrm{R}$ & Least \\
\hline
\end{tabular}




\begin{tabular}{|c|c|c|c|c|c|c|c|}
\hline & & & $\begin{array}{l}\text { Wren } \\
\text { Warbler }\end{array}$ & & & & Concern \\
\hline 121 & Cisticolidae & $\begin{array}{l}\text { Orthotomus } \\
\text { sutorius }\end{array}$ & $\begin{array}{l}\text { Indian } \\
\text { Tailor Bird }\end{array}$ & I & $\mathrm{C}$ & $\mathrm{R}$ & $\begin{array}{l}\text { Least } \\
\text { Concern }\end{array}$ \\
\hline 122 & Acrocephalidae & $\begin{array}{l}\text { Acrocephalus } \\
\text { dumetorum }\end{array}$ & $\begin{array}{l}\text { Blyth's } \\
\text { Reed } \\
\text { Warbler }\end{array}$ & I & $\mathrm{C}$ & $\mathrm{R}$ & $\begin{array}{l}\text { Least } \\
\text { Concern }\end{array}$ \\
\hline 123 & Turdidae & Geokichal citrine & $\begin{array}{l}\text { Orange } \\
\text { headed } \\
\text { Thrush }\end{array}$ & I & $\mathrm{C}$ & $\mathrm{R}$ & $\begin{array}{l}\text { Least } \\
\text { Concern }\end{array}$ \\
\hline 124 & Stenostiridae & $\begin{array}{l}\text { Culicicapa } \\
\text { ceylonensis }\end{array}$ & $\begin{array}{l}\text { Grey headed } \\
\text { Flycatcher }\end{array}$ & I & $\mathrm{Ra}$ & $\mathrm{R}$ & $\begin{array}{l}\text { Least } \\
\text { Concern }\end{array}$ \\
\hline 125 & Rhipiduridae & $\begin{array}{l}\text { Rhipidura } \\
\text { albicollis }\end{array}$ & $\begin{array}{l}\text { White } \\
\text { throated } \\
\text { Fantail }\end{array}$ & I & $\mathrm{Ra}$ & $\mathrm{R}$ & $\begin{array}{l}\text { Least } \\
\text { Concern }\end{array}$ \\
\hline 126 & Paridae & $\begin{array}{l}\text { Machlolophus } \\
\text { spilonotus }\end{array}$ & $\begin{array}{l}\text { Yellow } \\
\text { cheeked Tit }\end{array}$ & I & $\mathrm{C}$ & $\mathrm{R}$ & $\begin{array}{l}\text { Least } \\
\text { Concern }\end{array}$ \\
\hline 127 & Sittidae & $\begin{array}{l}\text { Sitta } \\
\text { cinnamoventris }\end{array}$ & $\begin{array}{l}\text { Chestnut } \\
\text { bellied } \\
\text { Nuthatch }\end{array}$ & $\mathrm{O}$ & $\mathrm{C}$ & $\mathrm{R}$ & $\begin{array}{l}\text { Least } \\
\text { Concern }\end{array}$ \\
\hline 128 & Motacillidae & Anthus trivialis & $\begin{array}{l}\text { Indian Tree } \\
\text { Pipit }\end{array}$ & I & $\mathrm{C}$ & $\mathrm{R}$ & $\begin{array}{l}\text { Least } \\
\text { Concern }\end{array}$ \\
\hline 129 & Motacillidae & Motacilla cinerea & $\begin{array}{l}\text { Grey } \\
\text { Wagtail }\end{array}$ & I & $\mathrm{C}$ & $\mathrm{M}$ & $\begin{array}{l}\text { Least } \\
\text { Concern }\end{array}$ \\
\hline 130 & Motacillidae & $\begin{array}{l}\text { Motacilla } \\
\text { maderaspatensis }\end{array}$ & $\begin{array}{l}\text { Large pied } \\
\text { Wagtail }\end{array}$ & I & $\mathrm{C}$ & $\mathrm{R}$ & $\begin{array}{l}\text { Least } \\
\text { Concern }\end{array}$ \\
\hline 131 & Motacillidae & Motacilla flava & $\begin{array}{l}\text { Yellow } \\
\text { Wagtail }\end{array}$ & I & $\mathrm{C}$ & $\mathrm{M}$ & $\begin{array}{l}\text { Least } \\
\text { Concern }\end{array}$ \\
\hline 132 & Motacillidae & Motacilla alba & $\begin{array}{l}\text { White } \\
\text { Wagtail }\end{array}$ & I & $\mathrm{C}$ & $\mathrm{M}$ & $\begin{array}{l}\text { Least } \\
\text { Concern }\end{array}$ \\
\hline 133 & Motacillidae & $\begin{array}{l}\text { Dendronanthus } \\
\text { indicus }\end{array}$ & $\begin{array}{l}\text { Forest } \\
\text { Wagtail }\end{array}$ & I & $\mathrm{C}$ & $\mathrm{M}$ & $\begin{array}{l}\text { Least } \\
\text { Concern }\end{array}$ \\
\hline 134 & Dicaeidae & Dicaeum agile & $\begin{array}{l}\text { Indian thick } \\
\text { billed } \\
\text { Flower } \\
\text { Pecker }\end{array}$ & $\mathrm{N}$ & $\mathrm{C}$ & $\bar{R}$ & $\begin{array}{l}\text { Least } \\
\text { Concern }\end{array}$ \\
\hline 135 & Nectariniidae & $\begin{array}{l}\text { Leptocoma } \\
\text { zeylonica }\end{array}$ & $\begin{array}{l}\text { Indian } \\
\text { purple } \\
\text { rumped } \\
\text { Sunbird }\end{array}$ & $\mathrm{N}$ & $\mathrm{C}$ & $\mathrm{R}$ & $\begin{array}{l}\text { Least } \\
\text { Concern }\end{array}$ \\
\hline 136 & Nectariniidae & $\begin{array}{l}\text { Cinnyris } \\
\text { jugularis }\end{array}$ & $\begin{array}{l}\text { Yellow } \\
\text { bellied } \\
\text { Sunbird }\end{array}$ & $\mathrm{N}$ & UC & $\mathrm{R}$ & $\begin{array}{l}\text { Least } \\
\text { Concern }\end{array}$ \\
\hline 137 & Nectariniidae & $\begin{array}{l}\text { Cinnyris } \\
\text { asiaticus }\end{array}$ & $\begin{array}{l}\text { Purple } \\
\text { Sunbird }\end{array}$ & $\mathrm{N}$ & $\mathrm{C}$ & $\mathrm{R}$ & $\begin{array}{l}\text { Least } \\
\text { Concern }\end{array}$ \\
\hline 138 & Nectariniidae & $\begin{array}{l}\text { Arachnothera } \\
\text { longirostra }\end{array}$ & $\begin{array}{l}\text { Little Spider } \\
\text { Hunter }\end{array}$ & $\mathrm{I}, \mathrm{N}$ & UC & $\mathrm{R}$ & $\begin{array}{l}\text { Least } \\
\text { Concern }\end{array}$ \\
\hline 139 & Zosteropidae & $\begin{array}{l}\text { Zosterops } \\
\text { palpebrosus }\end{array}$ & $\begin{array}{l}\text { Indian } \\
\text { White Eye }\end{array}$ & $\mathrm{I}, \mathrm{N}$ & $\mathrm{UC}$ & $\mathrm{R}$ & $\begin{array}{l}\text { Least } \\
\text { Concern }\end{array}$ \\
\hline 140 & Passeridae & $\begin{array}{l}\text { Passer } \\
\text { domesticus }\end{array}$ & $\begin{array}{l}\text { House } \\
\text { Sparrow }\end{array}$ & $\mathrm{G}$ & $\mathrm{C}$ & $\mathrm{R}$ & $\begin{array}{l}\text { Least } \\
\text { Concern }\end{array}$ \\
\hline 141 & Ploceidae & $\begin{array}{l}\text { Ploceus } \\
\text { philippinus }\end{array}$ & $\begin{array}{l}\text { Baya } \\
\text { Weaver }\end{array}$ & G,I & $\mathrm{C}$ & $\mathrm{R}$ & $\begin{array}{l}\text { Least } \\
\text { Concern }\end{array}$ \\
\hline 142 & Estrildidae & $\begin{array}{l}\text { Amandava } \\
\text { Formosa }\end{array}$ & $\begin{array}{l}\text { Green } \\
\text { Munia }\end{array}$ & G & $\mathrm{UC}$ & $\mathrm{R}$ & Vulnerable \\
\hline
\end{tabular}




\begin{tabular}{|l|l|l|l|l|l|l|l|}
\hline 143 & Estrildidae & Lonchura striata & $\begin{array}{l}\text { White } \\
\text { backed } \\
\text { Munia }\end{array}$ & G & C & R & $\begin{array}{l}\text { Least } \\
\text { Concern }\end{array}$ \\
\hline 144 & Estrildidae & $\begin{array}{l}\text { Lonchura } \\
\text { punctulata }\end{array}$ & $\begin{array}{l}\text { Scaly } \\
\text { breasted } \\
\text { Munia }\end{array}$ & G & C & R & $\begin{array}{l}\text { Least } \\
\text { Concern }\end{array}$ \\
\hline 145 & Fringillidae & $\begin{array}{l}\text { Carpodacus } \\
\text { erythrinus }\end{array}$ & $\begin{array}{l}\text { Indian Rose } \\
\text { Finch }\end{array}$ & G,I,N & C & R & $\begin{array}{l}\text { Least } \\
\text { Concern }\end{array}$ \\
\hline 146 & Phasianidae & Pavo cristatus & $\begin{array}{l}\text { Common } \\
\text { Peafowl }\end{array}$ & O & C & R & $\begin{array}{l}\text { Least } \\
\text { Concern }\end{array}$ \\
\hline 147 & Phasianidae & Gallus gallus & $\begin{array}{l}\text { Red Jungle } \\
\text { Fowl }\end{array}$ & O & C & R & $\begin{array}{l}\text { Least } \\
\text { Concern }\end{array}$ \\
\hline 149 & Phasianidae & $\begin{array}{l}\text { Francolinus } \\
\text { pondicerianus }\end{array}$ & $\begin{array}{l}\text { Indian grey } \\
\text { Francolin }\end{array}$ & G & Ra & R & $\begin{array}{l}\text { Least } \\
\text { Concern }\end{array}$ \\
\hline 150 & Turnicidae & Coturnix coturnix & $\begin{array}{l}\text { Common } \\
\text { Quail }\end{array}$ & G,I & C & R & $\begin{array}{l}\text { Least } \\
\text { Concern }\end{array}$ \\
\hline
\end{tabular}

Table 2:-Avifaunal distribution based on percentage occurrence in families

\begin{tabular}{|c|c|c|}
\hline SL NO & FAMILIES OF BIRDS RECORDED & PERCENTAGE OCCURRENCE \\
\hline 1 & Podicipedidae & 0.66 \\
\hline 2 & Anantidae & 1.33 \\
\hline 3 & Rallidae & 2.66 \\
\hline 4 & Jacanidae & 1.33 \\
\hline 5 & Charadriidae & 2 \\
\hline 6 & Phalacrocoracidae & 1.33 \\
\hline 7 & Ardeidae & 6 \\
\hline 8 & Ciconiidae & 0.66 \\
\hline 9 & Threskiornithidae & 0.66 \\
\hline 10 & Columbidae & 4.66 \\
\hline 11 & Psittaculidae & 2 \\
\hline 12 & Cuculidae & 3.33 \\
\hline 13 & Accipitridae & 6 \\
\hline 14 & Falconidae & 1.33 \\
\hline 15 & Strigidae & 4 \\
\hline 16 & Tytonidae & 0.66 \\
\hline 17 & Caprimuligidae & 1.33 \\
\hline 18 & Alcedenidae & 2.66 \\
\hline 19 & Meropidae & 2 \\
\hline 20 & Megalaimidae & 2 \\
\hline 21 & Coraciidae & 0.66 \\
\hline 22 & Upupidae & 0.66 \\
\hline 23 & Picidae & 3.33 \\
\hline 24 & Bucerotidae & 1.33 \\
\hline 25 & Hirundinidae & 2.66 \\
\hline 26 & Alaudidae & 0.66 \\
\hline 27 & Timaliidae & 1.33 \\
\hline 28 & Dicruridae & 2.66 \\
\hline 29 & Sturnidae & 4 \\
\hline 30 & Corvidae & 2 \\
\hline 31 & Tephrodornithidae & 0.66 \\
\hline 32 & Campephagidae & 1.33 \\
\hline 33 & Aegithinidae & 0.66 \\
\hline
\end{tabular}




\begin{tabular}{|l|l|l|}
\hline 34 & Chloropseidae & 1.33 \\
\hline 35 & Pycnonotidae & 2 \\
\hline 36 & Pellornidae & 0.66 \\
\hline 37 & Sylviidae & 0.66 \\
\hline 38 & Leiothrichidae & 0.66 \\
\hline 39 & Musicapidae & 5.33 \\
\hline 40 & Cisticolidae & 1.33 \\
\hline 41 & Acrocephalidae & 0.66 \\
\hline 42 & Turdidae & 0.66 \\
\hline 43 & Stenostridae & 0.66 \\
\hline 44 & Rhipiduridae & 0.66 \\
\hline 45 & Paridae & 0.66 \\
\hline 46 & Sittidae & 0.66 \\
\hline 47 & Motacillidae & 4 \\
\hline 48 & Dicaeidae & 0.66 \\
\hline 49 & Nectariniidae & 2.66 \\
\hline 50 & Zosteropidae & 0.66 \\
\hline 51 & Passeridae & 0.66 \\
\hline 52 & Ploceidae & 0.66 \\
\hline 53 & Estrildidae & 2 \\
\hline 54 & Fringillidae & 0.66 \\
\hline 55 & Phasianidae & 2.66 \\
\hline 56 & Turnicidae & 0.66 \\
\hline
\end{tabular}

Abbreviations: R- Resident, M- Migratory, C- Common, UC- Uncommon, Ra- Rare, LC- Least Concern, NTNear Threatened, VU-Vulrenable, EN-Endangered, I- Insectivores, P- Piscivores, Ca- Carnivores, O- Omnivores, FFrugivores, G- Granivores, N- Nectarivores
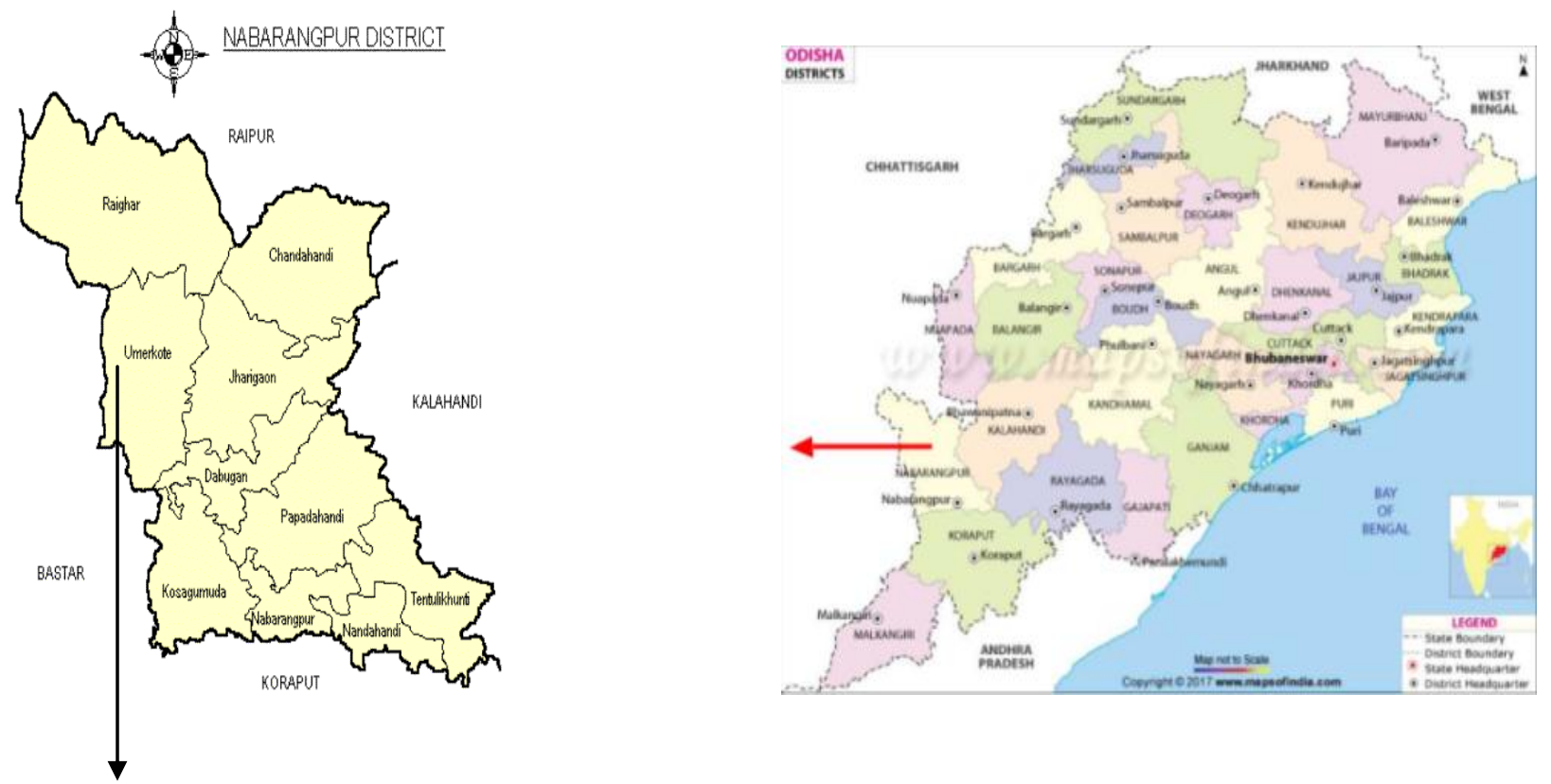


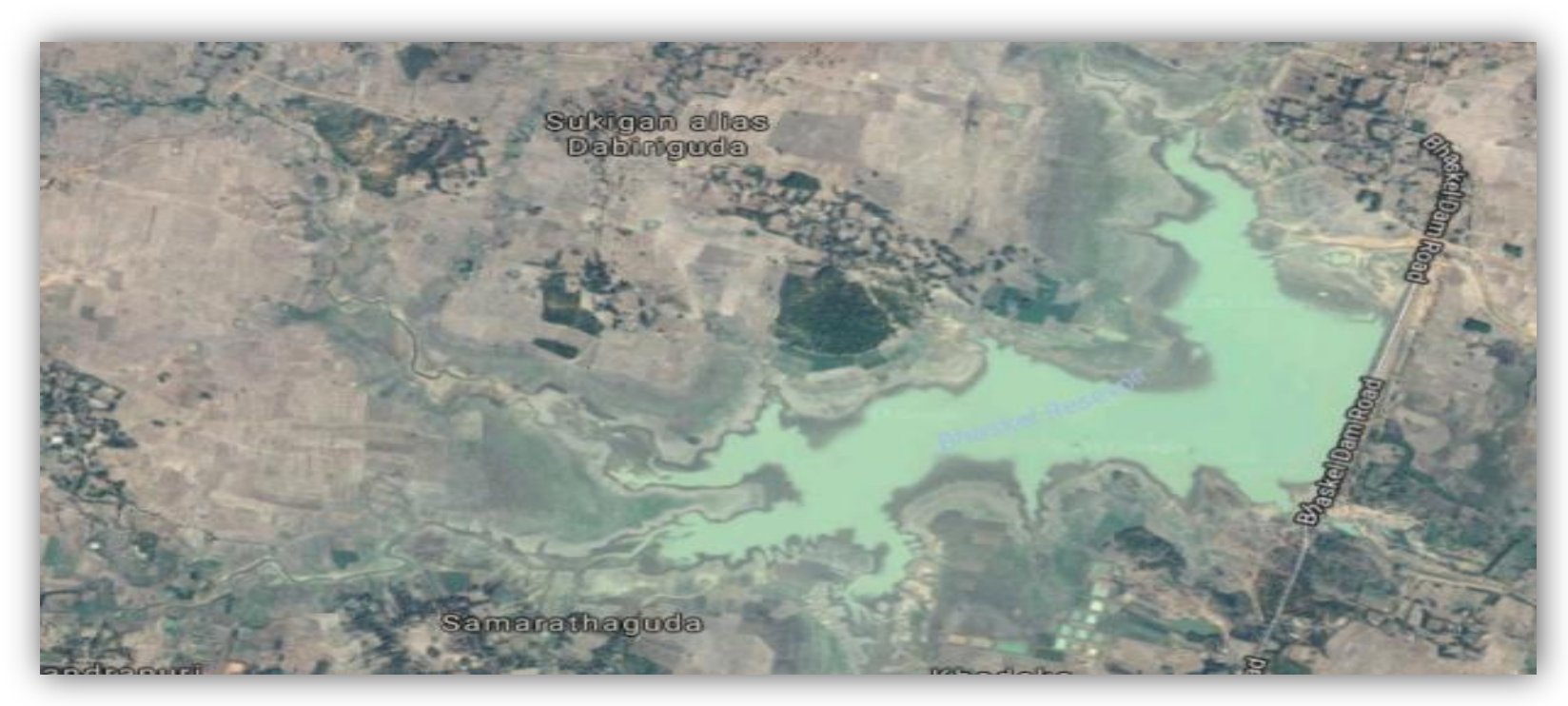

Fig 1:- Location map showing Bhaskel Dam Reservoir in Umerkote town, Nabarangpur district of Odisha.

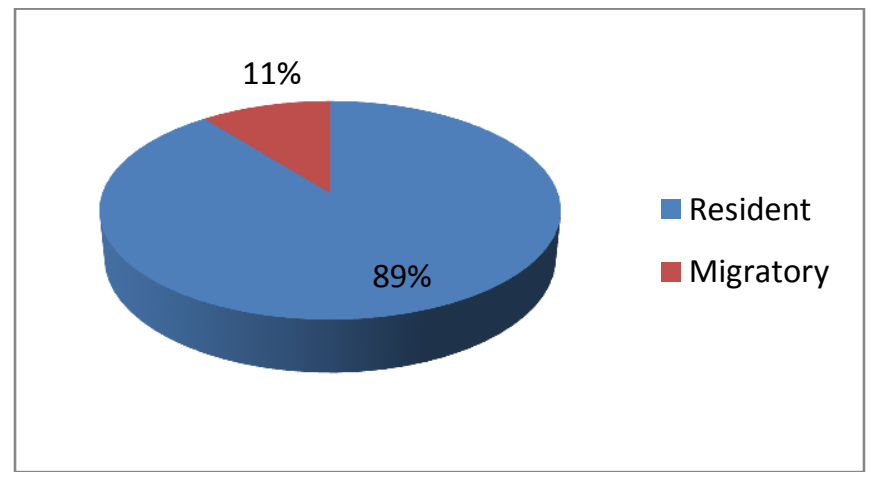

Fig 2:-Avifaunal distribution (in percent) based on abundance

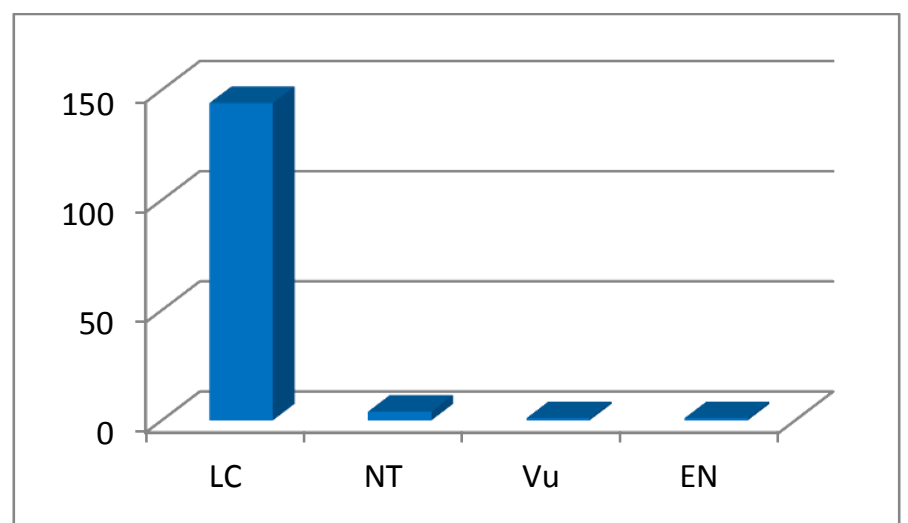

Fig 3:-Avifaunal distribution based on IUCN category 


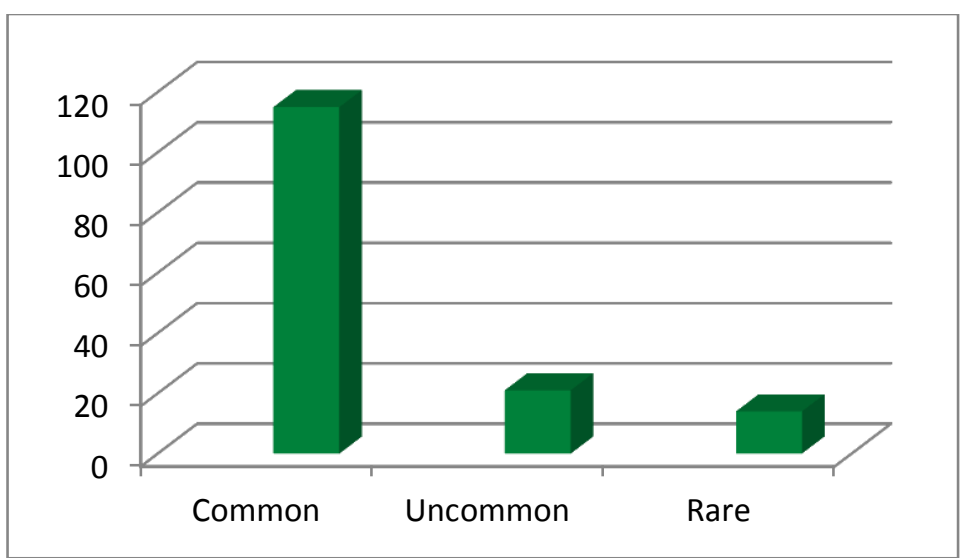

Fig 4:-Avifaunal distribution based on status

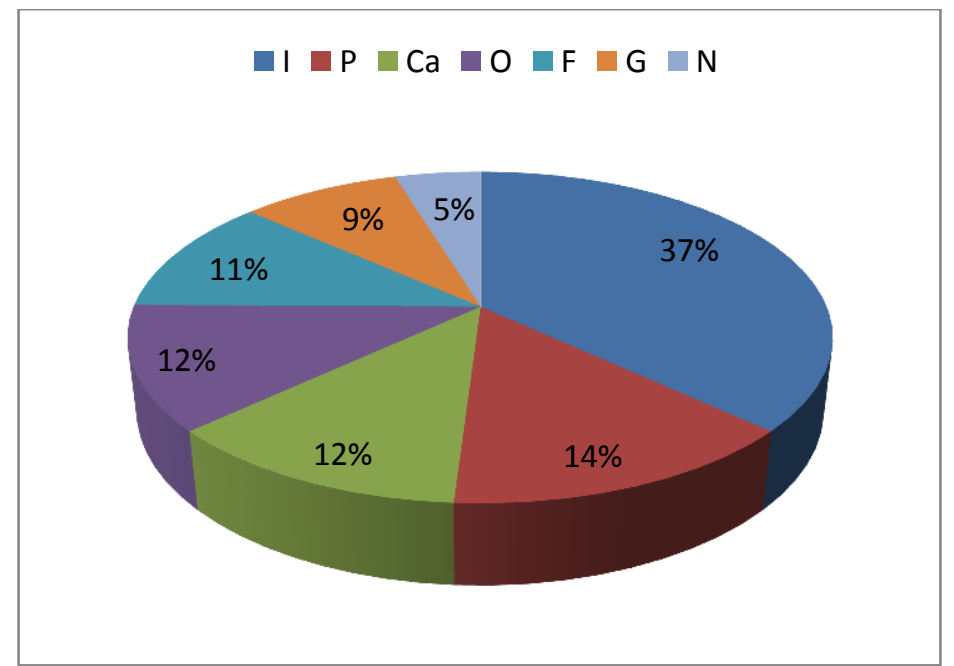

Fig 5:-Avifaunal distribution (in percent) based on dietary pattern

\section{Conclusion:-}

The present study which recorded 150 species of birds reflects a moderately healthy overall biodiversity for the study location. But it must be mentioned that the study location under present investigation are facing anthropogenic disturbances in the forms of urbanization, mining activities, livelihood dependence (mainly in the form of cattle grazing and fuel wood collection). To add salt to the wound poaching of birds is a major issue for this area like most other parts of India. Natural calamities like forest fire also have disastrous effects on wildlife from the present study location. To conclude it may be noted that the area was studied for short time span, a more intensive study would surely result in identifying more bird species. The impact of anthropogenic alteration of the habitats in and around the present study location also needs intensive studies.

\section{Acknowledgements:-}

The authors are grateful to all the staffs of the forest department of Nabarangpur district and also to various residents of Umerkote town and Nabarangpur district for their cooperation and help during the study period.

\section{References:-}

1. Abdulali, H., (1984). Seasonality and occurrence of birds in the Eastern Ghats. J. Bombay Nat. Hist. Soc. 81 (1): 191.

2. Ali, S., (2002). The Book of Indian Birds (13th revised edition) Oxford University Press, New Delhi. 
3. Ali, S. and Ripley, S.D., (1987). Compact handbook of the birds of India and Pakistan. Bombay: Oxford University Press.

4. Das, S. K., Dash, N., Ahmed, R. A., \& Debata, S., (2010). Birds of North Orissa University campus at the base of Similipal Biosphere Reserve, Orissa, India. Newsletter for Birdwatchers 50 (2): 25-29.

5. Dev. U.N., (1997). A profile of Birds of Orissa, Reference Orissa, A.N. Tiwari, Enterprising Pub., 596 p.

6. Gopi, G. V., \&Pandav, B., (2007). Avifauna of Bhitarkanika mangroves, India. Zoos' Print Journal 22 (10): 2839-2847, 2847i-ii.

7. Manakadan, R. and Pittie, A. (2001). Standardized common and Scientific names of the birds of the Indian Sub Continent. Buceros. 6(1): 1-37.

8. Mukherjee, A. K., (1952). On a collection of birds from the Simlipal Hills, Mayurbhanj District, Orissa. Records of the Indian Museum 50 (2): 157-172.

9. Newton, I., (1995). The contribution of some recent research on birds to ecological understanding. J. Anim. Ecol., 64, 675-696.

10. Ripley, S. D., (1979). Changes in the bird fauna of a forest area; Simlipal Hills, Mayurbhanj District, and Dhenkanal District, Orissa. J. Bombay Nat. Hist. Soc. 75 (3): 570-574.

11. Sahu, H. K. \& Kar, S. K., (1999). Study in population status of aquatic birds in Nalaban sanctuary, Chilika lake, Orissa. Pranikee. 19 \& 20: 11-15.

12. Sahu, H. K., \& Rout, S. D., (2005). Checklist of waterbirds in Mayurbhanj district, Orissa. Zoos' Print Journal 20 (9): 1992-1993. 